\title{
LONGITUDINAL MYELITIS: RARE NEUROLOGICAL MANIFESTATION OF LUPUS
}

Patrícia Milani de Moraes ${ }^{1, \star}$, Renata Vaz de Oliveira ${ }^{1}$, Carlos Augusto Rodrigues Padilha ${ }^{1}$, Maria Juliana da Silva Almeida ${ }^{1}$, lane Tamara Dondé ${ }^{1}$, Gustavo Roberto Lourenço ${ }^{1}$, Danielly Dantas Pimentel ${ }^{1}$, Juliana de Jesus Boscolo ${ }^{1}$

1.Faculdade de Medicina de São José do Rio Preto, São José do Rio Preto (SP), Brazil.

*Corresponding author: pat_jau@hotmail.com

\section{BACKGROUND}

Systemic lupus erythematosus (SLE) can present with neurological impairments, such as acute confusional state, cerebrovascular disease, seizures, peripheral neuropathies, and myelopathies. We will report a rare case of longitudinal myelitis as a sign of lupus activity.

\section{CASE REPORT}

A 30-year-old woman with lupus was admitted with a history of odynophagia for 30 days, evolving after 10 days, with ascending and progressive paresis of the lower limbs, urinary incontinence, and oral thrush. On physical examination, there was grade 0 strength in the lower limbs and decreased sensitivity. After 2 days, there was also loss of strength in the upper limbs (grade 2 strength) but without dysphagia and dyspnea. Complementary tests: anti-DNA: $400 \mathrm{U} / \mathrm{mL}$, complement (C3: $56 \mathrm{mg} / \mathrm{dL}$ and C4: $4 \mathrm{mg} / \mathrm{dL}$ ). Liquor collection performed (leukocytes: $247 / \mathrm{mm}^{3}$, neutrophils: $61 \%$, protein: $139 \mathrm{mg} / \mathrm{dL}$, lymphocytes: $27 \%$, monocytes: $12 \%$ and glucose: $35 \mathrm{mg} / \mathrm{dL}$ ). Negative liquor bacteria culture. Negative serologies. Neuroaxis magnetic resonance showed extensive longitudinal hypersignal lesions from the bulbospinal transition to the level of the medullary cone. After excluding the differential diagnoses, treatment for longitudinal myelitis was started as a form of lupus activity, with 5 days of pulse therapy with methylprednisolone and after 5 days of plasmapheresis. During treatment, the patient partially recovered strength in the upper limbs (grade IV strength) and grade 3 strength in the left lower limb and grade 2 strength in the right lower limb.

\section{CONCLUSION}

Longitudinal myelitis is a rare manifestation ( $1 \%$ to $2 \%$ of patients with SLE), affecting the motor, sensory and autonomic systems, and although it usually presents during the acute phase of the disease, 1/3 of the cases can happen outside periods of activity, therefore it should be treated quickly for better outcome. Treatment options include pulse therapy with methylprednisolone, plasma exchange and cyclophosphamide. Among poor prognostic factors, we can mention: limb flaccidity, urinary incontinence and delay in the beginning of the treatment. Although the patient had limb flaccidity and urinary incontinence, there was a rapid improvement in strength, especially in the upper limbs. She continues to await the end of plasmapheresis.

\section{KEYWORDS}

Systemic lupus erythematosus, Longitudinal myelitis, Methylprednisolone, Plasmapheresis. 